\title{
Improved DC Performance Analysis of a Novel Asymmetric Extended Source Tunnel FET for Fast Switching Application
}

RITAM DUTTA ( $\square$ ritamdutta1986@gmail.com )

Sikkim Manipal Institute of Technology https://orcid.org/0000-0003-3089-778X

T.D. Subash

Mangalam College of Engineering

Nitai Paitya

Sikkim Manipal Institute of Technology

\section{Research Article}

Keywords: AES-TFET, Surface potential distribution, Electric field variation, BTBT, TCAD, Numerical modelling

Posted Date: March 30th, 2021

DOl: https://doi.org/10.21203/rs.3.rs-347124/v1

License: (c) (i) This work is licensed under a Creative Commons Attribution 4.0 International License. Read Full License 


\title{
Improved DC Performance Analysis of a Novel Asymmetric Extended Source Tunnel FET for Fast Switching Application
}

\author{
Ritam Dutta ${ }^{1,3}$. T. D. Subash ${ }^{2}$. Nitai Paitya ${ }^{3}$ \\ Received: 19 March 2021 / Accepted: XXXXXXXXXXXX \\ (C) Springer Nature B.V. 2021
}

\begin{abstract}
A two-dimensional analytical model for asymmetric extended source tunnel field effect transistor (AES-TFET) has been developed to obtain better device performance. The proposed device model has been analytically modelled and performed by solving 2-D Poisson's equation. Surface potential distribution, electric field variation and band-to-band tunneling (BTBT) rate have been investigated by this numerical modelling. The source region of novel structure of TFET has been extended (varied $2 \mathrm{~nm}$ to $6 \mathrm{~nm}$ ) to incorporate corner effect, which allows BTBT through a thin tunneling barrier, with controlled ambipolar conduction. This eventually produces better source-channel interface tunneling for a $n$-channel AES-TFET. 2-D numerical device simulator (SILVACO TCAD) has been used for simulation work. The simulated work has been finally validated by analytical modelling of AES-TFET. Better I $\mathrm{IN}_{\mathrm{ON}} \mathrm{I}_{\mathrm{OFF}}$ and switching ratio has been obtained from this novel TFET structure.
\end{abstract}

Keywords AES-TFET ' Surface potential distribution · Electric field variation · BTBT $\cdot$ TCAD $\cdot$ Numerical modelling.

\section{Introduction}

With the advent of nanoscience and nanotechnology in nano-scaled devices, the physical sizes of transistors have been scaled down categorically. By following the Moore's law prediction in 2022, the miniaturization has reached its limit for metal oxide filed effect transistors (MOSFETs) [1]. In this connection, various scaling issues has been emerged in last two decades viz. short channel effects (SCE), drain induced barrier lowering (DIBL) [2]. To overcome these issues, continuous research is going on in novel structures of MOSFETs. But in present scenario, the limited subthreshold swing (SS) of MOSFETs at around $60 \mathrm{mV} /$ decade has been a major drawback for researchers.

Ritam Dutta

ritamdutta1986@gmail.com

T. D. Subash

tdsubash2007@gmail.com

Nitai Paitya

nitai.p@smit.smu.edu.in

1 Department of Electronics and Comm. Engineering, Surendra Institute of Engineering \& Management, West Bengal734009, India

2 Department of Electrical and Electronics Engineering, Mangalam College of Engineering, Kerala -686631, India.

3 Department of Computer Sc. and Engineering, Sikkim Manipal Institute of Technology, Sikkim-737136, India
Keeping all device parameter scaling issues in mind, a new device physics has been inculcated in nanodevice engineering. In this case quantum tunneling has been introduced as a solid replacement of earlier FETs [3]. The typical energy band structure with ultra-thin barrier helped researchers to develop tunneling junction devices (TJD) using band to band tunneling (BTBT) phenomenon. In this paper, prior to this work, several literatures were surveyed based on structural and material engineering [4-14]. The effect of homogeneous and heterogeneous material in tunneling junctions [15], effect of pocket intrinsic doping on single as well as multi gate tunneling FETs [16-17], effect of device performance based on various high- $k$ materials [18], stress-strain effects in source-channel ( $n$-channel) and drain-channel ( $p$-channel) TJDs [19], usage of carbon nano-tubes (CNT) in tunneling FETs [20], nano-wire tunneling FETs [21], capacitive effects in modified TJD structures [22], various symmetric and asymmetric tunneling device structures has been studied to meet the earlier mentioned scaling issues and device performance factors.

In our paper the typical extended design of source region has been modelled to introduce corner effect in source-channel junction for $n$-channel TFET. The entire device structure details have been mentioned in section 2 , where the typical device parameters are used based on recent established literature survey. The device physics based analytical modelling has been introduced in section 3. Here the two-dimensional Poisson's equation is used for numerical analysis. Parabolic approximation method is considered for potential distribution analysis across the 
channet region. In section 4, simulation and analytical results and discussion are compared for validation of our work. The energy band diagram analysis, surface potential distribution, electric field variation, transfer characteristics and transconductance analysis are graphically plotted. Finally, in section 5, the conclusion and future scope of our proposed AES-TFET structure has been discussed.

\section{Device Structure}

In this section, a typical extended design of source region has been modelled to introduce corner effect in sourcechannel junction for $n$-channel TFET (figure 1). Unlike conventional symmetric single gate tunnel FET (figure 2 ), the asymmetric extended source results better BTBT tumneting across ultra-thin tumneting barrier. The tunneling region is divided into five parts. Region 1 (length of $\mathrm{R}_{1}, \mathrm{~L}_{1}=5 \mathrm{~nm}$ ) is made of $\mathrm{Si}-\mathrm{Ge}$ material for better tunneling, Region 2 (length of $\mathrm{R}_{2}, \mathrm{~L}_{2}=10 \mathrm{~nm}$ ), Region 3 (length of $\mathrm{R}_{3}, \mathrm{~L}_{3}=20 \mathrm{~nm}$ ) and Region 4 (length of $\mathrm{R}_{4}, \mathrm{~L}_{4}=5 \mathrm{~nm}$ ) to consider the potential distribution at drain side depletion region. Region 1 and 2 are considered unitedly as Region 5 (length of $\mathrm{R}_{5}, \mathrm{~L}_{1}+\mathrm{L}_{2}=\mathrm{L}_{5}=15 \mathrm{~nm}$ ). $\mathrm{P}^{+}$source side doping $\left(N_{\mathrm{S}}\right)=10^{20} \mathrm{~cm}^{-3}$, intrinsic channel doping $\left(N_{\mathrm{ch}}\right)=10^{14} \mathrm{~cm}^{-3}$ and $\mathrm{N}^{+}$drain side doping $\left(N_{\mathrm{D}}\right)=$ $10^{18} \mathrm{~cm}^{-3}$, body thickness $\left(t_{\mathrm{Si}}\right)=10 \mathrm{~nm}$ and gate oxide thickness $\left(t_{\mathrm{ox}}\right)=2 \mathrm{~nm}$ is considered in our simulation work.

Fig. 1. 3-D view of proposed AES-TFET device model

Fig. 2. 3-D view of symmetric conventional TFET device model
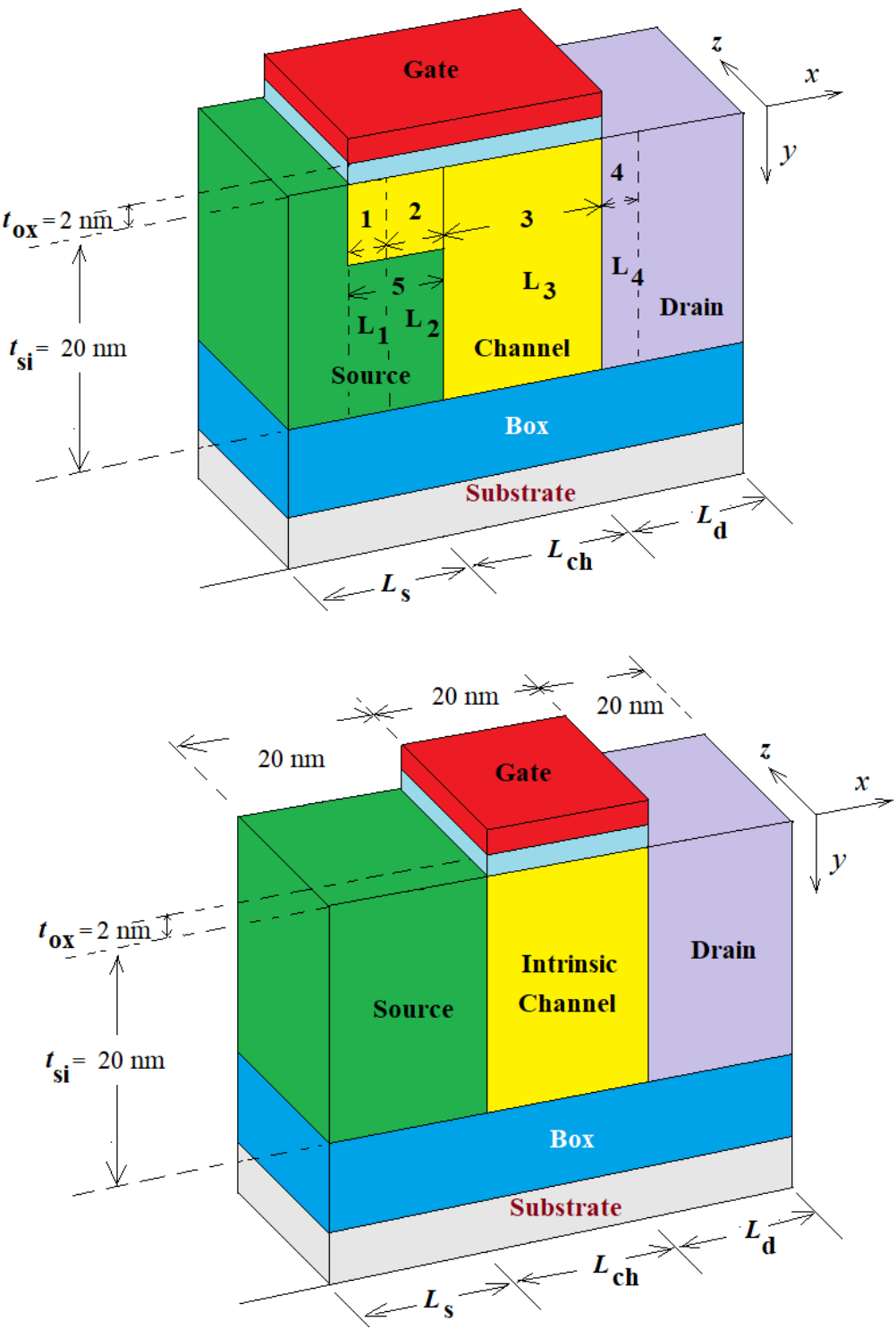


\section{Analytical Model}

In order to investigate the electrostatic potential across the identified regions from $R_{1}$ to $R_{4}$ (figure 1 ), two-dimensional Poisson's equation is used as follows:

$$
\begin{aligned}
\frac{\partial^{2} \psi_{i}}{\partial x^{2}}+\frac{\partial^{2} \psi_{i}}{\partial y^{2}}=\frac{q N_{i}}{\varepsilon_{i}} \quad \text { for } i \\
=1,2,3,4 \quad 0 \leq x \\
\leq L \text { and } 0 \leq y \leq t_{S i}
\end{aligned}
$$

Where, $\psi_{i}$ is electrostatic potential for all four regions $\left(\mathrm{R}_{1}\right.$ to $\mathrm{R}_{4}$ ), $q$ is charge, $N_{i}$ is doping concentration of respective regions, $\varepsilon_{i}$ is the dielectric constant. $\mathrm{L}$ is assumed as overall length of all four regions i.e. $\mathrm{L}=\mathrm{L}_{1}+\mathrm{L}_{2}+\mathrm{L}_{3}+\mathrm{L}_{4}$. The channel thickness is denoted as $t_{S i}$, used for numerical analysis.

Here in our work, parabolic approximation is assumed for solving the surface potential distribution across the regions. So, we can write:

$$
\psi_{i}(x, y)=a_{0 i}(x)+a_{1 i}(x) y+a_{2 i}(x) y^{2}
$$

Where, constant coefficients are denoted as $a_{0 \mathrm{i}}, a_{1 \mathrm{i}}$ and $a_{2 \mathrm{i}}$ which are the arbitrary functions of $x$. Now after assuming the arbitrary coefficients for all regions along $x$ axis, their values can be calculated using following boundary conditions. So, from the planar device structure, it can be clearly understandable that the boundary conditions are completely based on continuity of surface potential profile and electric field distribution profile. Therefore, from gate oxide layer to semiconductor layer i.e. $\left(\mathrm{SiO}_{2}\right.$ to $\mathrm{Si}$ interface) the boundary conditions are set as $y=0$ and $y=t_{\mathrm{si}}$.

$$
\begin{aligned}
\left.\psi_{i}(x, y)\right|_{y=0} & =\psi_{S i}(x) \\
\left.\frac{\partial \psi_{i}(x, y)}{\partial y}\right|_{y=0} & =\frac{C_{o x}}{\varepsilon_{i}}\left(\psi_{S i}(x)-V_{G}\right) \\
\left.\frac{\partial \psi_{i}(x, y)}{\partial y}\right|_{y=0}= & 0
\end{aligned}
$$

Where, $\psi_{S i}(x)$ is denoted for surface potential along lateral direction i.e. ( $x$ axis) and $C_{o x}$ is denoted as gate oxide capacitance.

Here in our device model, the fringing field at channel-drain interface depletion region (i.e. region $\mathrm{R} 4$ ) has been considered. Therefore, gate oxide thickness $\left(C_{o x}\right)$ in equation 4 can be replaced by $\frac{2}{\pi} C_{o x}[23]$ and effective gate voltage $\left(V_{G}\right)$ can also be written as:

$$
\begin{aligned}
V_{G} & =V_{g s}-V_{f b i} \\
V_{f b i} & =V_{g s}-\phi_{m}+\chi_{i}+\frac{E_{g i}}{2}+\phi_{f i}
\end{aligned}
$$

Where, $\phi_{m}$ is metal work function and $\phi_{f i}$ is denoted as fermi potential. $V_{f b i}$ is denoted as flat band voltage, $\chi_{i}$ and $\mathrm{E}_{\mathrm{gi}}$ represents electron affinity and energy band gap of respective identified regions. In the region 1 , in order to introduce stress - strain effect; $\mathrm{Si}$-Ge combination is introduced. Due to which the band gap energy of region $\mathrm{R}_{1}$ (i.e. $E_{g 1}$ ) can be written as [24]:

$$
\begin{aligned}
& E_{g 1}=1.10-0.34 x \quad \text { where, } x=\text { mole } \\
& \text { fraction of } G e
\end{aligned}
$$

Now using the above-mentioned boundary conditions equation 3 to 5 and equations 6 to 7 , the co-efficient (equation 2) can be estimated as:

$$
\begin{aligned}
& a_{0 i}(x)=\psi_{S i}(x) \\
& a_{1 i}(x)=\frac{\varepsilon_{o x}}{\varepsilon_{i} t_{o x}}\left(\psi_{S i}(x)-V_{G}\right) \\
& a_{2 i}(x)=\frac{\varepsilon_{o x}}{2 \varepsilon_{i} t_{o x} t_{s}}\left(V_{G}-\psi_{S i}(x)\right)
\end{aligned}
$$

Therefore, substituting these coefficient values from equation 9, 10 and 11 in equation 1 ; the surface potential from the regions $\mathrm{R}_{1}$ to $\mathrm{R}_{4}$ can be written as:

$\psi_{S i}^{\prime \prime}(x)-k_{i}^{2}(x) \psi_{S i}(x)=k_{i}^{2} \eta_{i}$

Where, $k_{i}=\sqrt{\frac{C_{O x}}{t_{S i} \varepsilon_{i}}}$ and $\eta_{i}=\frac{q N_{i}}{\varepsilon_{i} k_{i}^{2}}-V_{G}$

\section{So, in general the equation 11 can be written} as:

$$
\psi_{S i}(x)=A_{i} e^{\left(k_{i} x_{i}\right)}+B_{i} e^{\left(-k_{i} x_{i}\right)}-\eta_{i}
$$

Since, the device model is designed assuming n-channel AESTFET, therefore the band-to-band tunneling regions i.e. $\mathrm{R}_{1}$ and $\mathrm{R}_{2}$ regions combinedly denoted as region $5\left(\mathrm{R}_{5}\right)$. Using Poisson's equation, we can write:

$\frac{\partial^{2} \psi_{s 5}}{\partial x^{2}}=\frac{q N_{2}}{\varepsilon_{2}}$

Therefore, similarly the equation 13 can be written in general:

$\psi_{s 5}(x, y)=\frac{q \cdot N_{2}}{\varepsilon_{2}} x^{2}+C_{51} x+C_{52}$

Finally, all these coefficients $\mathrm{A}_{\mathrm{i}}, B_{\mathrm{i}}, C_{51}$ and $C_{52}$ can be solved using following boundary conditions. Here, the $\mathrm{x}$ is replaced by respective region lengths $L_{1}$ to $L_{4}$.

$$
\begin{gathered}
V_{b i s}=\psi_{s 1}(0)=-\frac{\delta T}{q} \ln \left(\frac{N_{1}}{N_{2}}\right) \\
\psi_{s 1}\left(L_{1}\right)=\psi_{s 2}\left(L_{1}\right) \\
\therefore \frac{\partial \psi_{s 1}\left(L_{1}\right)}{\partial x}=\frac{\partial \psi_{s 2}\left(L_{1}\right)}{\partial x}
\end{gathered}
$$




$$
\begin{aligned}
& \psi_{s 2}\left(L_{2}\right)=\psi_{s 3}\left(L_{2}\right) \\
& \therefore \frac{\partial \psi_{s 2}\left(L_{2}\right)}{\partial x}=\frac{\partial \psi_{s 3}\left(L_{2}\right)}{\partial x} \\
& \psi_{s 3}\left(L_{3}\right)=\psi_{s 4}\left(L_{3}\right) \\
& \therefore \frac{\partial \psi_{s 3}\left(L_{3}\right)}{\partial x}=\frac{\partial \psi_{s 4}\left(L_{3}\right)}{\partial x} \\
& \text { Since, } \psi_{s 5}\left(L_{1}+L_{2}\right)=\psi_{s 5}\left(L_{3}\right) \\
& \therefore \frac{\partial \psi_{s 5}\left(L_{1}+L_{2}\right)}{\partial x}=\frac{\partial \psi_{s 5}\left(L_{3}\right)}{\partial x}
\end{aligned}
$$

Similarly, for region 4 at channel-drain interface, the drainbias potential can be written as:

$$
\begin{aligned}
& V_{b i d}=\psi_{s 4}\left(L_{4}\right)=V_{d s}+\frac{\delta T}{q} \ln \left(\frac{N_{3}}{N_{2}}\right) \\
& \therefore L_{4}=\sqrt{\frac{2 \varepsilon_{i} N_{2}\left(V_{G}-V_{d s}-V_{b i d}\right)}{q N_{3}\left(N_{2}+N_{3}\right)}}
\end{aligned}
$$

Now, from equation 13,15 and (16 to 26), the coefficients $\mathrm{A}_{\mathrm{i}}, B_{\mathrm{i}}, C_{51}$ and $C_{52}$ can be calculated as follows:

$$
\begin{gathered}
C_{1}=A_{4}\left[Z_{1}\left(k_{3}+k_{4}\right) e^{\left(k_{4} L_{3}\right)}\right. \\
-Z_{2}\left(k_{3}\right. \\
\left.\left.-k_{4}\right) e^{\left(k_{4} L_{3}\right)}\right] \\
+B_{4}\left[Z_{1}\left(k_{3}-k_{4}\right) e^{\left(-k_{4} L_{3}\right)}\right. \\
-Z_{2}\left(k_{3}\right. \\
\left.\left.+k_{4}\right) e^{\left(-k_{4} L_{3}\right)}\right] \\
+\left[Z_{1} k_{3}\left(\eta_{3}-\eta_{4}\right)-Z_{2} k_{3}\left(\eta_{3}-\right.\right. \\
\left.\left.\eta_{4}\right)-2 L_{2} \frac{q N_{5}}{\varepsilon_{i}}\right] \\
\begin{array}{c}
C_{2}=A_{4}\left[D_{2} R_{1}+D_{3} R_{2}\right] \\
+ \\
+B_{4}\left[D_{2} R_{3}+D_{3} R_{4}\right] \\
-\left[D_{2} R_{5}+D_{3} R_{5}\right.
\end{array} \\
\begin{array}{c}
\left.B_{4}=\quad D_{1}\right] \\
\left\{\left(V_{b i s}+\eta_{1}\right) e^{\left(k_{4} L_{4}\right)}\right\}-\left\{\left(V_{b i d}+V_{d s}+\eta_{4}\right)\left(E_{1}+E_{3}\right)\right\}
\end{array} \\
\frac{-\left\{\left(G_{1}+G_{2}\right) e^{\left(k_{4} L_{4}\right)}\right\}}{\left(E_{2}+E_{4}\right) e^{\left(k_{4} L_{4}\right)}-\left(E_{1}+E_{3}\right) e^{\left(-k_{4} L_{4}\right)}} \\
\begin{array}{c}
A_{4}=\quad \\
\left\{\left(V_{b i s}+\eta_{1}\right) e^{\left(-k_{4} L_{4}\right)}\right\}-\left\{\left(V_{b i d}+V_{d s}+\eta_{4}\right)\left(E_{2}+E_{4}\right)\right\} \\
\frac{-\left\{\left(G_{1}+G_{2}\right) e^{\left(-k_{4} L_{4}\right)}\right\}}{\left(E_{1}+E_{3}\right) e^{\left(-k_{4} L_{4}\right)}-\left(E_{2}+E_{4}\right) e^{\left(k_{4} L_{4}\right)}}
\end{array}
\end{gathered}
$$

$$
\begin{aligned}
& A_{3}=\frac{1}{2 k_{3} e^{\left(k_{3} L_{3}\right)}} \mid A_{4}\left(k_{3}+\right. \\
& \left.k_{4}\right) e^{\left(k_{4} L_{3}\right)}+B_{4}\left(k_{3}-k_{4}\right) e^{\left(-k_{4} L_{3}\right)}+ \\
& \left.k_{3}\left(\eta_{3}-\eta_{4}\right)\right] \\
& B_{3}=\frac{1}{2 k_{3} e^{\left(-k_{3} L_{3}\right)}}\left[A _ { 4 } \left(k_{3}-\right.\right. \\
& \left.k_{4}\right) e^{\left(k_{4} L_{3}\right)}+B_{4}\left(k_{3}+k_{4}\right) e^{\left(-k_{4} L_{3}\right)}+ \\
& \left.k_{3}\left(\eta_{3}-\eta_{4}\right)\right] \\
& A_{2}=A_{4}\left[Y_{1}\left(k_{3}+k_{4}\right) e^{\left(k_{4} L_{3}\right)}\right. \\
& +Y_{2}\left(k_{3}\right. \\
& \left.\left.-k_{4}\right) e^{\left(k_{4} L_{3}\right)}\right] \\
& +B_{4}\left[Y_{1}\left(k_{3}-k_{4}\right) e^{\left(-k_{4} L_{3}\right)}\right. \\
& +Y_{2}\left(k_{3}\right. \\
& \left.\left.+k_{4}\right) e^{\left(-k_{4} L_{3}\right)}\right] \\
& +\frac{1}{4 k_{2} e^{\left(k_{2} L_{2}\right)}}\left[O_{6}\left(k_{2}+k_{3}\right)+O_{7}\left(k_{2}-\right.\right. \\
& \left.\left.k_{3}\right)+O_{8}\right] \\
& B_{2}=A_{4}\left[W_{1}\left(k_{3}+k_{4}\right) e^{\left(k_{4} L_{3}\right)}\right. \\
& +W_{2}\left(k_{3}\right. \\
& \left.\left.-k_{4}\right) e^{\left(k_{4} L_{3}\right)}\right] \\
& +B_{4}\left[W_{1}\left(k_{3}-k_{4}\right) e^{\left(-k_{4} L_{3}\right)}\right. \\
& +W_{2}\left(k_{3}\right. \\
& \left.\left.+k_{4}\right) e^{\left(-k_{4} L_{3}\right)}\right] \\
& +\frac{1}{4 k_{2} e^{\left(-k_{2} L_{2}\right)}}\left[O_{6}\left(k_{2}-k_{3}\right)+O_{7}\left(k_{2}+\right.\right. \\
& \left.\left.k_{3}\right)+O_{8}\right] \\
& A_{1}=A_{4} E_{1}+B_{4} E_{2}+G_{1} \\
& B_{1}=A_{4} E_{3}+B_{4} E_{4}+G_{2}
\end{aligned}
$$

Now, by differentiating the surface potential functions obtained from above numerical analysis; the lateral as well as vertical electric field can be achieved. Therefore, along ( $x-$ axis) channel length, the lateral e-filed can be obtained as follows:

$$
\begin{aligned}
& E_{x i}=-k_{i}\left[A_{i} e^{\left(k_{i} x_{i}\right)}-B_{i} e^{\left(-k_{i} x_{i}\right)}\right] \\
& \text { for Region } R_{1} \text { to } R_{4} \\
& E_{x 5}=-\left[2 \frac{q N_{2}}{\varepsilon_{2}} L_{2}+C_{1}\right]
\end{aligned}
$$

Similarly, the vertical e-filed along ( $y$ - axis) also can be achieved as follows:

$$
\begin{aligned}
& E_{y i}=-\left[A_{1 i}(x)+2 y a_{2 i}(x)\right] \\
& \text { for Region } R_{1} \text { to } R_{4} \\
& E_{y 5}=0
\end{aligned}
$$

In our work, a new carrier transport phenomenon is introduced 
i.e. band-to-band (BTBT) quantum tumneting. Since n-

channel AES-TFET is designed with introducing a thin $\left(\mathrm{L}_{1}=\right.$

$5 \mathrm{~nm}) \mathrm{Si}-\mathrm{Ge}$ material for better tunneling at source - channel interface, so the drain current $\left(I_{d}\right)$ can be obtained as:

$$
\begin{aligned}
& I_{d}=q \int_{L_{1}}^{L_{4}} G_{B T B T} d v \\
& \text { for Region } R_{1} \text { to } R_{4}
\end{aligned}
$$

$$
\text { So, } \quad G_{B T B T}=A \frac{\left|E^{2}\right|}{\sqrt{E_{g i}}} e^{\left(-B \frac{E_{g i}^{1.5}}{|E|}\right)}
$$

Where, $|E|=\left|\sqrt{E_{x}^{2}+E_{y}^{2}}\right|$

As mentioned in equation $42, \mathrm{~A}$ and $\mathrm{B}$ are the model parameters, whose values are considered as $4 \times 10^{14} \mathrm{~cm}^{-3} \mathrm{~s}^{-1}$ and $1.9 \times 10^{7} \mathrm{~V} \mathrm{~cm}^{-1}$ respectively. After obtaining the drain current $\left(I_{d}\right)$ i.e. ON-state current, the transconductance $\left(\mathrm{g}_{\mathrm{m}}\right)$ can also be calculated as: $g_{m}=\left(d I_{D} / d V_{G S}\right)_{V_{D S}}$

\section{Results and Discussion}

\subsection{Energy Band Diagram Analysis}

Minute study of energy band diagram helps to assess the carrier transport across source channel interface for a $n$ channel tunnel FET. Unlike conventional TFET structures, our proposed AES-TFET offers a typical extended source structure which eventually capitalizes the corner effect at the junction. Due this corner effect, the thin tunneling barrier can easily be tunnelled through by carriers from source to intrinsic channel regions of AES-TFET. Considering indirect BTBT tunneling and other device model parameters, the simulation work is performed using 2D SILVACO TCAD device simulator. The dotted encircled area shown in figure 3 represents the improved tunneling window developed by proposed AES-TFET model at $0.5 \mathrm{~V}$ of supply voltage.
Fig.3 Energy band diagram of AES-TFET at $\mathrm{V}_{\mathrm{gs}}=1 \mathrm{~V}, \mathrm{~V}_{\mathrm{DD}}=$ $0.5 \mathrm{~V}$

\subsection{Surface Potential Distribution}

The potential distribution along the surface of our proposed device AES-TFET structure is investigated based on the two-dimensional Poisson's equation. Across the source-channel interface region and due to its typical extended structure, the variation of surface potential is substantially plotted in figure 4 . The simulation is done using TCAD device simulator, keeping supply voltage $\left(\mathrm{V}_{\mathrm{DD}}\right)$ fixed at $0.5 \mathrm{~V}$ in order to utilize this device model for low power applications.

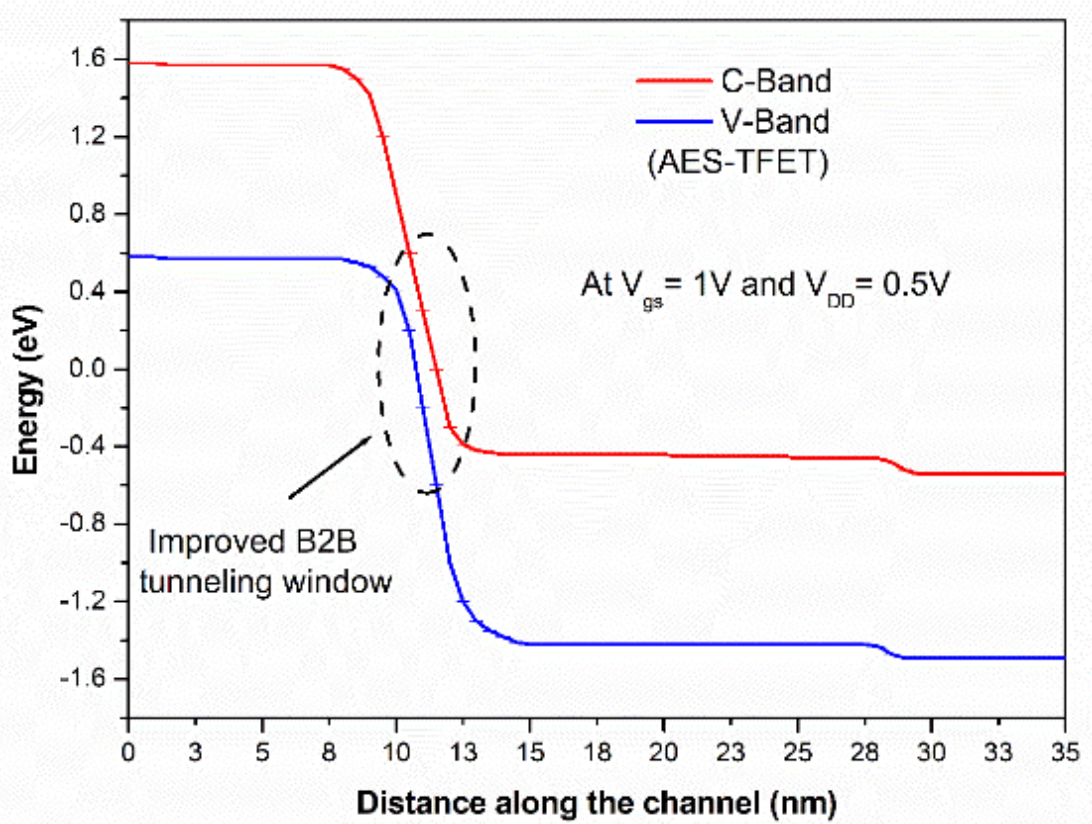

Therefore, by varying the gate-source voltage $\left(\mathrm{V}_{\mathrm{gs}}\right)$ ranging from $1 \mathrm{~V}$ to $0.5 \mathrm{~V}$, the potential distribution can be studied. In figure 4 , it can be very well observed that the simulation data become almost in line with analytical dataset. 


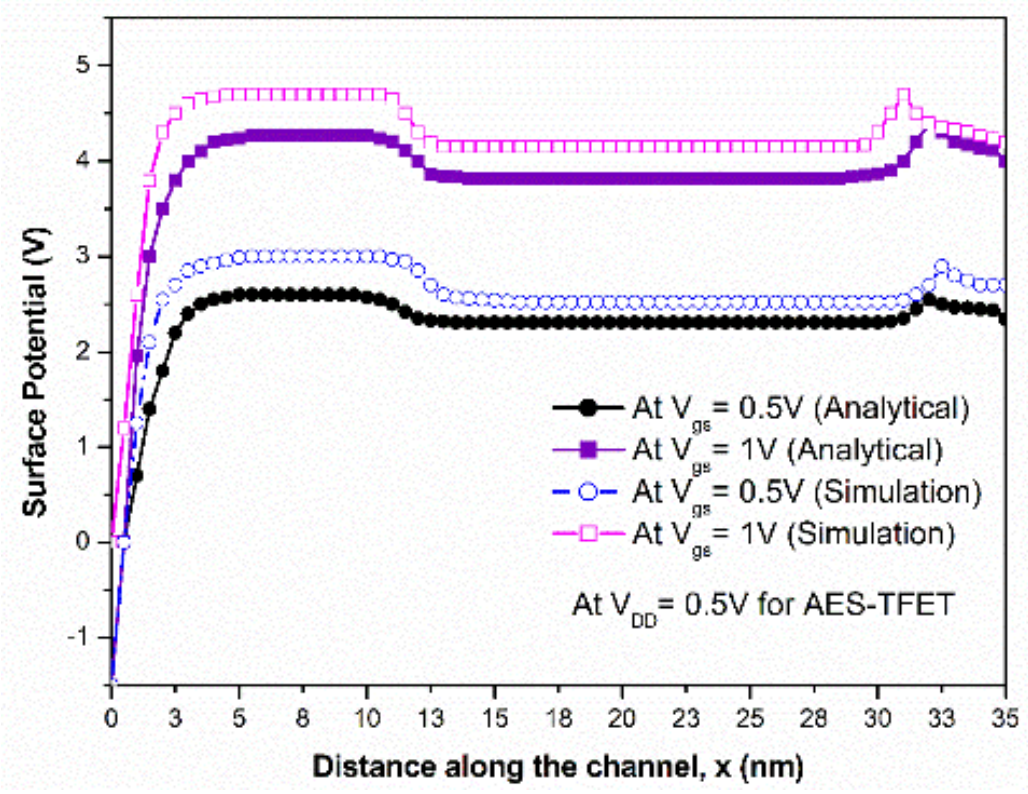

\subsection{Electric Field variation along the channel}

Figure 5 shows the comparison between simulation and analytical values obtained from electric field variation. For different gate to source voltages $\left(\mathrm{V}_{\mathrm{gs}}\right)$, the electric field (lateral and vertical) varies considerably. In our proposed structure, the source has been extended to introduce corner effect at source - channel tunneling interface. This further result large overlapping between the bottom edge of conduction band and top edge of valence band. Because of which, in this fig. 5, a spike has been identified at source-channel junction. However, electric field distribution remains same at rest of the channel region except a slight change near drain depleted region. Here, the analytical results almost match with the simulated graphical representations. This validates the work.
Fig.5 Electric field distribution along the channel of AES-TFET for different gate-to-source voltages $\left(\mathrm{V}_{\mathrm{gs}}\right)$ at $\mathrm{V}_{\mathrm{DD}}=0.5 \mathrm{~V}$

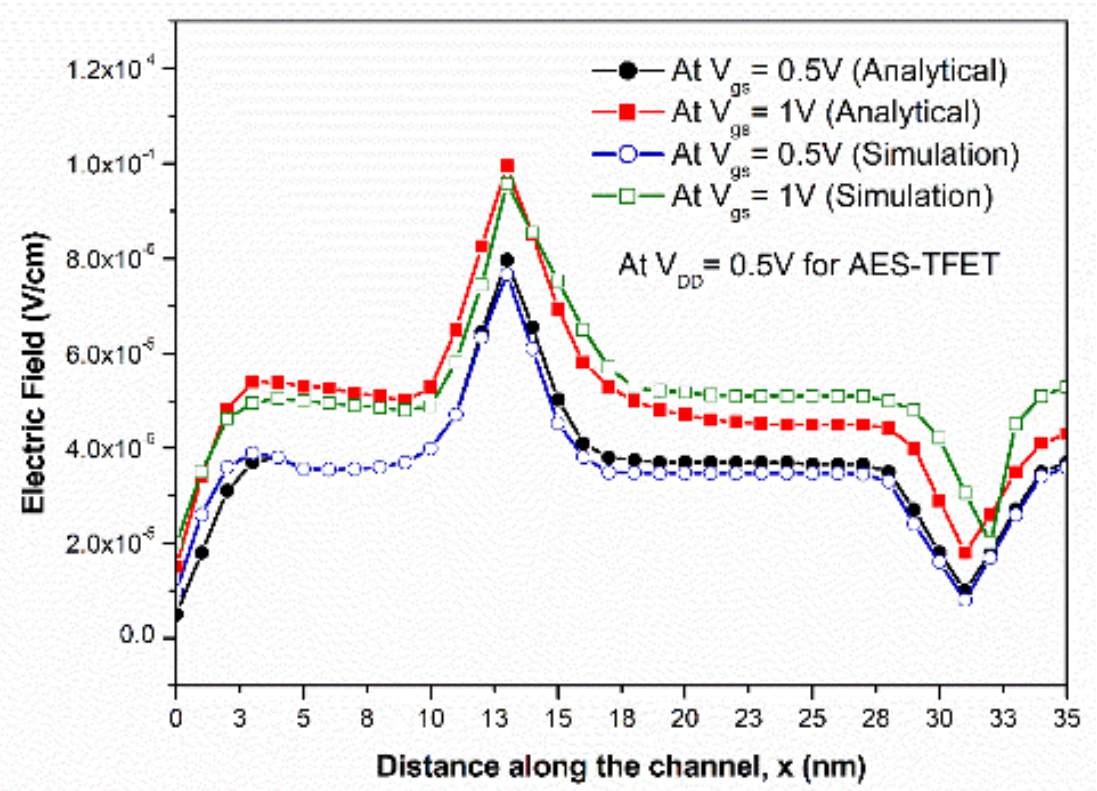




\subsection{Transfer Characteristics}

Although the tunnel FET structure with modified device models act as proper replacement of conventional MOSFET device structures, but the low ON state current has been major limitation in tunnel FET device modelling. Keeping these limitations in view, in this work the conventional source has been extended to introduce the sharp corner deep inside the lowly doped intrinsic channel. In addition to this, at this tunneling junction $\mathrm{Si}$-Ge material has been proposed to embed the stress-strain effect, which further helped to boost the drain current $\left(\mathrm{I}_{\mathrm{ON}}\right)$. In figure 6 , the transfer characteristics depicts the improvement in drain eurrent for better device performance.

\subsection{Transconductance}

Solving the equation 43 , the analytical modelling of proposed asymmetric extended source tunnel field effect transistor (AES-TFET) structure has been validated with simulation data of transconductance $\left(\mathrm{g}_{\mathrm{m}}\right)$. Further the transconductance of AES-TFET has been compared with conventional TFET symmetric structures (figure 7).
Fig.6 Transfer characteristics $\left(\mathrm{I}_{\mathrm{d}} \mathrm{vs} \mathrm{V}_{\mathrm{gs}}\right)$ analysis between Conventional TFET and proposed AES-TFET at $\mathrm{V}_{\mathrm{DD}}=0.5 \mathrm{~V}$
Fig.7 Transconductance ( $g_{\mathrm{m}}$ vs $\mathrm{V}_{\mathrm{gs}}$ ) analysis between Conventional TFET and proposed AES-TFET at $\mathrm{V}_{\mathrm{DD}}=0.5 \mathrm{~V}$
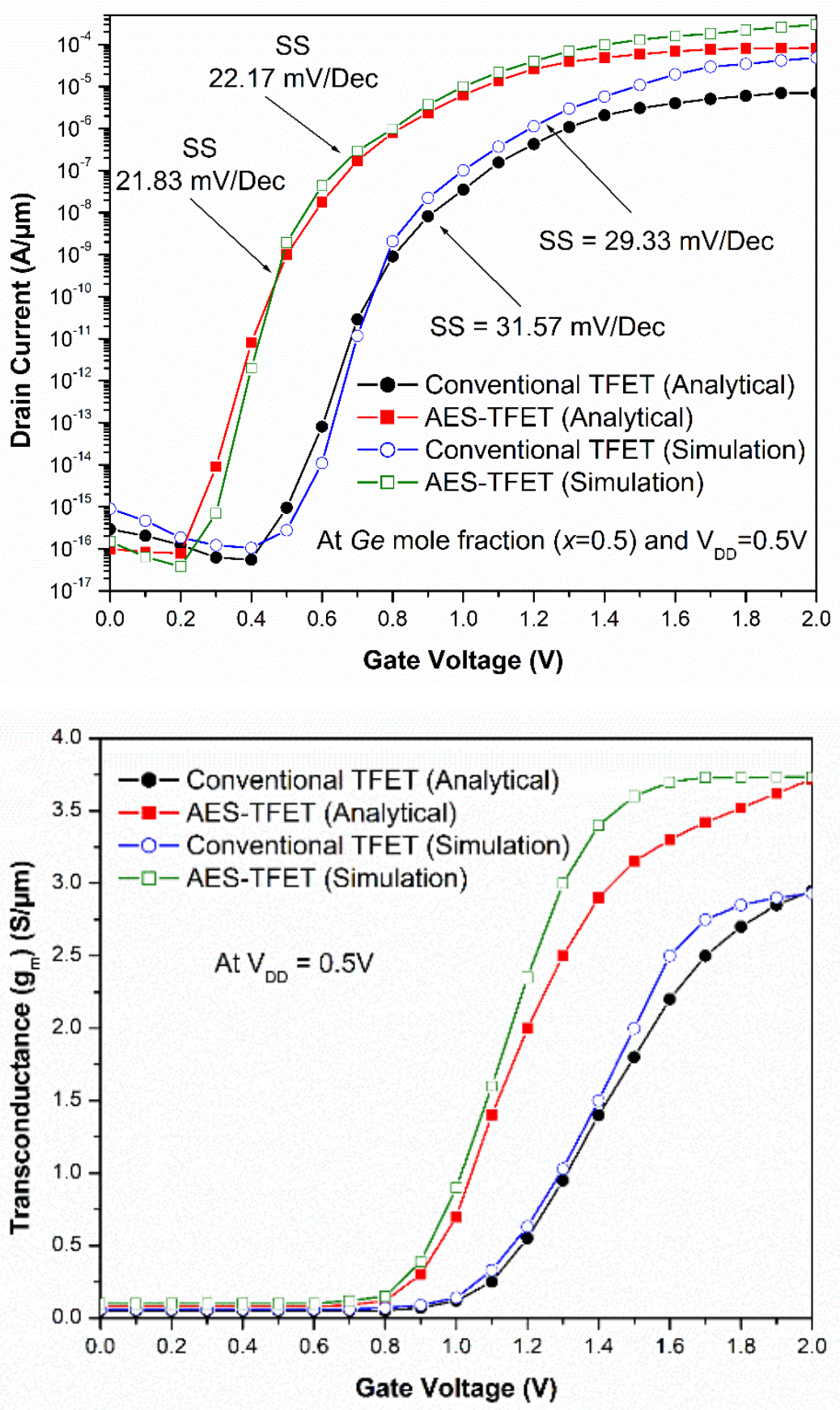


\section{Conclusion}

In this work a detail numerical analysis has been performed for our proposed asymmetric extended source tunnel field effect transistor (AES-TFET). Analytical modelling of potential distribution along the channel surface, electric field variation, drain current and transconductance has been derived using 2-D Poisson's equation, considering parabolic approximation method. Besides this, using 2-D SILVACO TCAD device simulator, all device characteristics has been simulated and graphically plotted. The analytical model shows a substantial agreement in matching with simulation model dataset. These graphical representations validate our work. All D.C characteristics has been performed at $0.5 \mathrm{~V}$ of supply voltage for low power device applications. The major challenge of improving sub-threshold swing (SS) has been taken care of by the proposed model, where SS $=21.83$ $\mathrm{mV} /$ decade has been recorded as best compared to symmetric single gated conventional TFET structure. The ON state current and ambipolar current suppression has also been achieved at greater extent, shown in transfer function analysis of both device models.

\section{Acknowledgement (Funding Statement)}

This work was supported in part by All India Council for Technical Education (AICTE) Govt. of India under Research Promotion Scheme for North-East Region (RPS-NER) vide ref: File No. 8-139/RIFD/RPS-NER/Policy-1/2018-19.

\section{Author Contributions}

The corresponding author has done all the literature survey, novel device modelling, mathematical analysis, the second author (Dr. T.D. Subash) and third author (Dr. N. Paitya) have guided their Ph.D. scholar Mr. Ritam Dutta in documentation work.

\section{Conflict of Interest}

The Authors declare that they have no conflict of interest.

\section{Ethics Declarations}

Not Applicable.

\section{Compliance with Ethical Standards}

Since there is no conflict of interest between the authors, and this research has not involved any human participants or animals, therefore this section is not applicable.

\section{Availability of Data and Material}

All related data and material have been included in the main manuscript referred from the reference section.

\section{Consent for Publication}

Since this work has entirely been performed by the research scholar Mr. R. Dutta, guided by the other two authors, therefore this section is not applicable.

\section{Consent to Participate}

Not Applicable.

\section{References}

1. Moore GE. (2006) Cramming More Components onto Integrated Circuits, IEEE Solid-State Circuits Society Newsletter. 11 (3):33-35.

2. Ionescu AM, Riel H. (2011) Tunnel field-effect transistors as energy efficient electronic switches, Nature. 479 (7373):329-337.

3. Seabaugh AC, Zhang Q. (2010) Low-voltage tunnel transistors for beyond CMOS logic. Proc. IEEE. 98(12):2095-2110.

4. Thomas N, Philip Wong HS. (2017) The end of Moore's Law: a new beginning for information technology, IEEE J. Comput. Sci. Eng. 19(2):41-50.

5. Turkane SM, Kureshi AK. (2016) Review of Tunnel Field Effect Transistor (TFET), International Journal of Applied Engineering, 11 (7):4922-4929.

6. Yousefi R, Shabani M. (2011) A model for carbon nanotube FETs in the ballistic limit, Microelectronics Journal. 42 (11):1299-1304.

7. Talukdar J, Rawat G, Choudhuri B, Signh K, Mummaneni K. (2019) A Novel Extended Source TFET with $\delta \mathrm{p}+-$ SiGe Layer, Silicon. doi: 10.1007/s12633-019-00321-3.

8. Shen M, Saikin S, Cheng MC. (2005) Spin injection in spin FETs using a step-doping profile, IEEE Transactions on Nanotechnology. 4 (1):4044.

9. Yoon JS, Kim K, Rim T, Baek CK. (2017) Performance and variations induced by single interface trap of nanowire FETs at 7-nm node, IEEE Transactions on Electron Devices. 64(2):339-345.

10. Das B, Bhowmick B. Noise behaviour of ferro electric tunnel FET, (2020) Microelectronics Journal. 96. doi: 10.1016/j.mejo.2019.104677.

11. Fiori G, Iannaccone G. (2007) Simulation of graphene nanoribbon fieldeffect transistors, IEEE Electron Device Letters. 28 (8):760-762.

12. Zhang Q, Zhao W, Seabaugh A. (2006) Low-Subthreshold-Swing Tunnel Transistors, IEEE Electron Device Letters. 27 (4):297-300.

13. Gonzalez R, Gordon BM, Horowitz MA. (1997) Supply and Threshold Voltage Scaling for Low Power CMOS, IEEE Journal of Solid-State Circuits. 32 (8):1210-1216.

14. Madan J, Chaujar R. (2017) Gate drain underlapped-PNIN-GAA-TFET for comprehensively upgraded analog/RF performance, Superlattices Microstructures. 102:17-26.

15. Ilatikhameneh H, Ameen TA, Klimeck G, Appenzeller J, Rahman R. (2015) Dielectric engineered tunnel field-effect transistor, IEEE Electron Device Lett. 36 (10):1097-1100.

16. Jhaveri R, Nagavarapu V, Woo JCS. (2011) Effect of Pocket Doping and Annealing Schemes on the Source-Pocket Tunnel Field-Effect Transistor, IEEE Transactions on Electron Devices. 58(1):80 - 86. doi: 10.1109/TED.2010.2089525

17. Das GD, Mishra GP, Dash S. (2018) Impact of source-pocket engineering on device performance of dielectric modulated tunnel FET, Superlattices and Microstructures. 124:131-138. doi: 10.1016/j.spmi.2018.10.014

18. Karbalaei M, Dideban D, Heidari H. (2020) Influence of high- $k$ insulator and source stack on the performance of a double gate tunnel FET: a simulation study. Journal of Computational Electronics. 19:1077-1084. doi: 10.1007/s10825-020-01497-3. 
19. TH J H design with dual-material source and compressed drain, Applied Physics A, 126 (81). doi: 10.1007/s00339-019-3266-5.

20. Xu L, Qiu C, Peng LM. (2020) Suppression of leakage current in carbon nanotube field-effect transistors. Nano Res. Doi:10.1007/s12274-020-3135-8

21. Sessi V, Simon M, Mulaosmanovic H, Pohl D, Loeffler M, Mauersberger T, Franz PG, Mikolajick T, Walter M, Weber A. (2020) Silicon Nanowire Ferroelectric Field-Effect Transistor. Advanced Elec. Materials. Doi: 10.1002/aelm.201901244

22. Dey A, Ray R. (2020) Tunneling Barrier Modulation in Negative Capacitance-TFET. Proc. of Int. Conf. on Frontiers in Computing and Systems. Advances in Intelligent Systems and Computing, 1255. Doi:10.1007/978-981-15-7834-2 76 .

23. Lin SC, Kuo JB. (2003) Modeling the Fringing Electric Field Effect on the Threshold Voltage of FD SOI nMOS Devices With the LDD/Sidewall Oxide Spacer Structure, IEEE Transactions on Electron Devices, 50 (12):2559 - 2564.

24. Talukdar J, Rawat G, Singh K, Mummaneni K. (2020) Comparative Analysis of the Effects of Trap Charges on Single- and Double-Gate Extended-Source Tunnel FET with dp+ Si-Ge Pocket Layer, Journal of Electronic Materials, 49(7):4333-4342. 
Figures

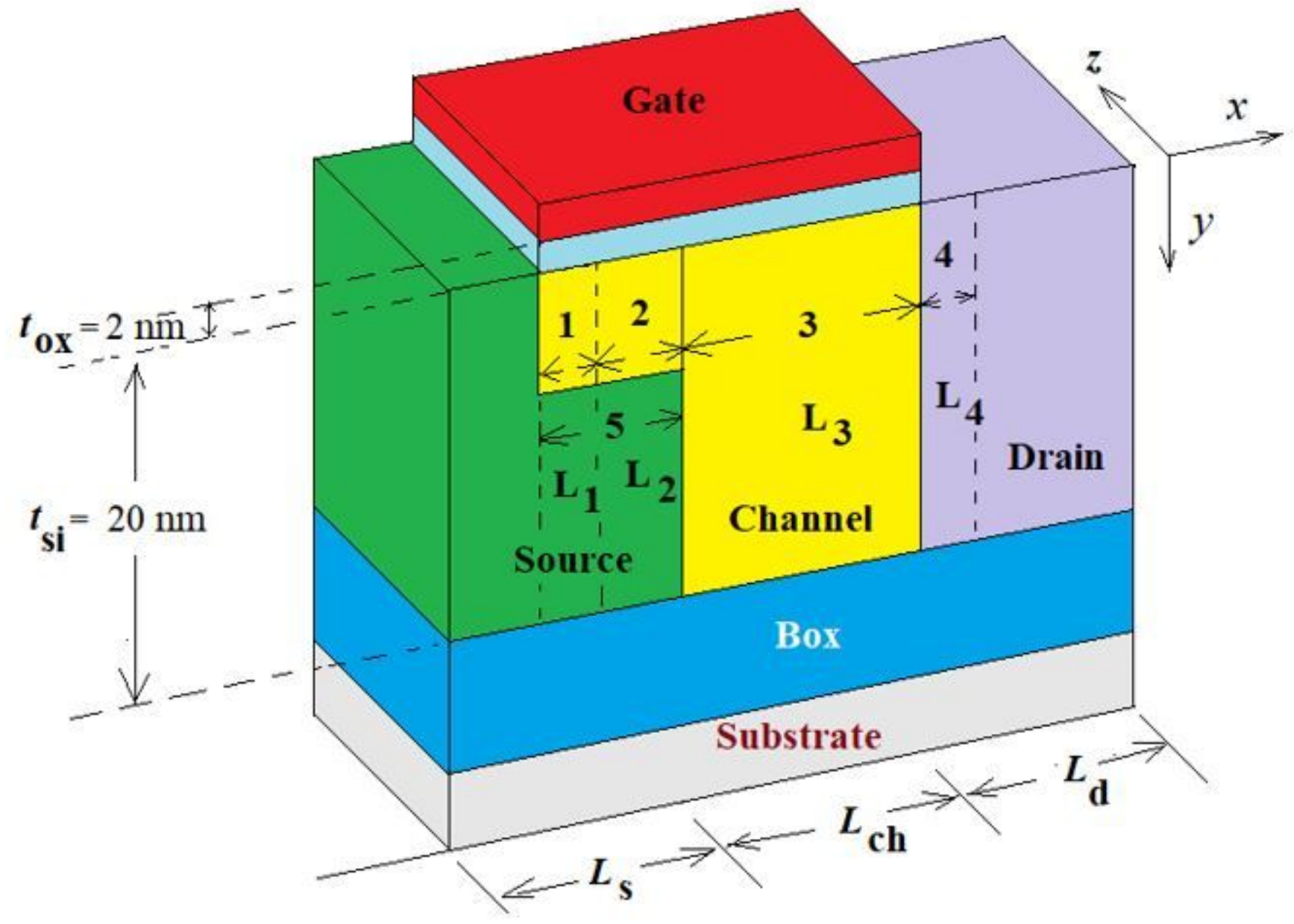

Figure 1

3-D view of proposed AES-TFET device model 


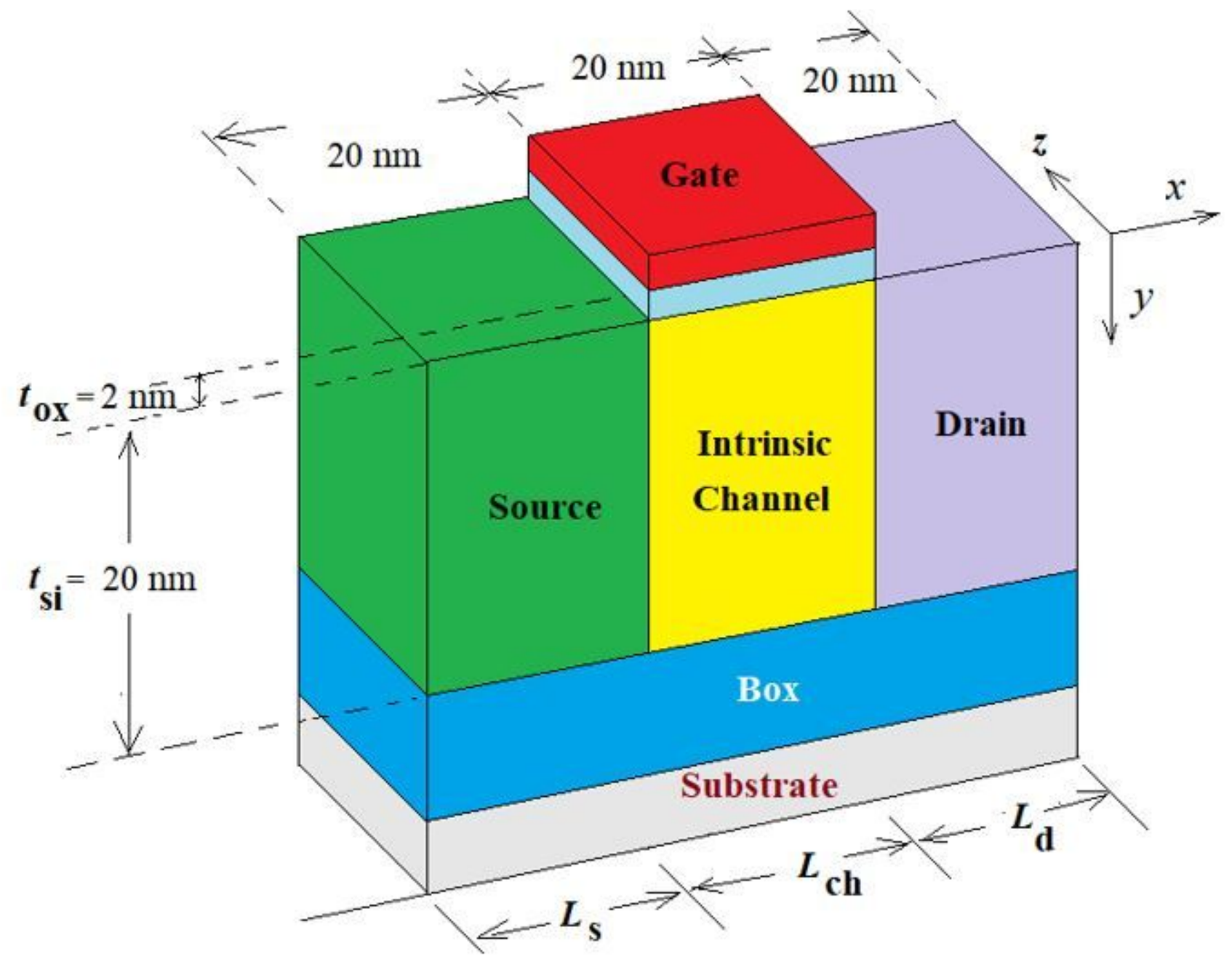

Figure 2

3-D view of symmetric conventional TFET device model 


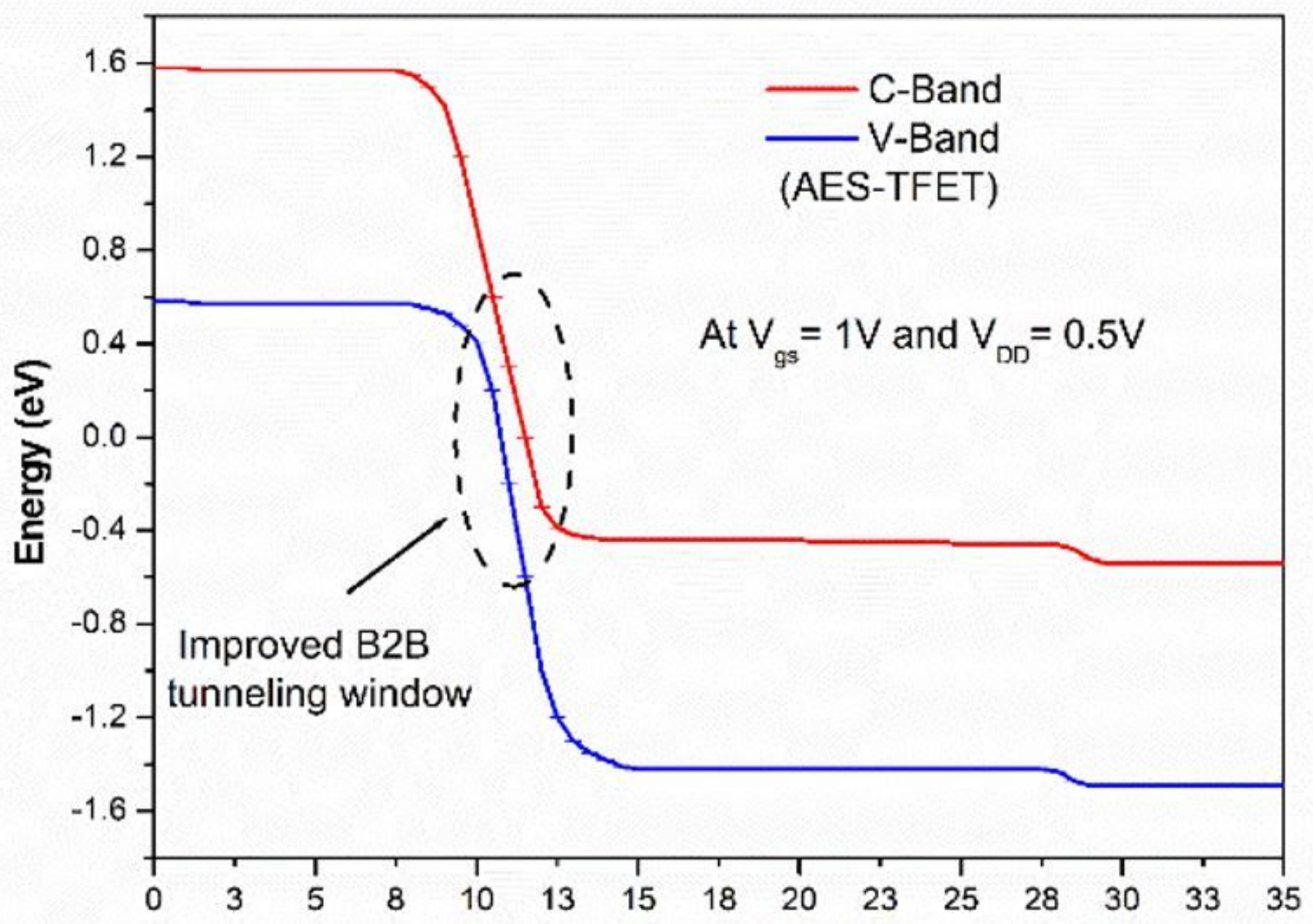

Figure 3

Energy band diagram of AES-TFET at $\mathrm{Vgs}=1 \mathrm{~V}, \mathrm{VDD}=0.5 \mathrm{~V}$ 


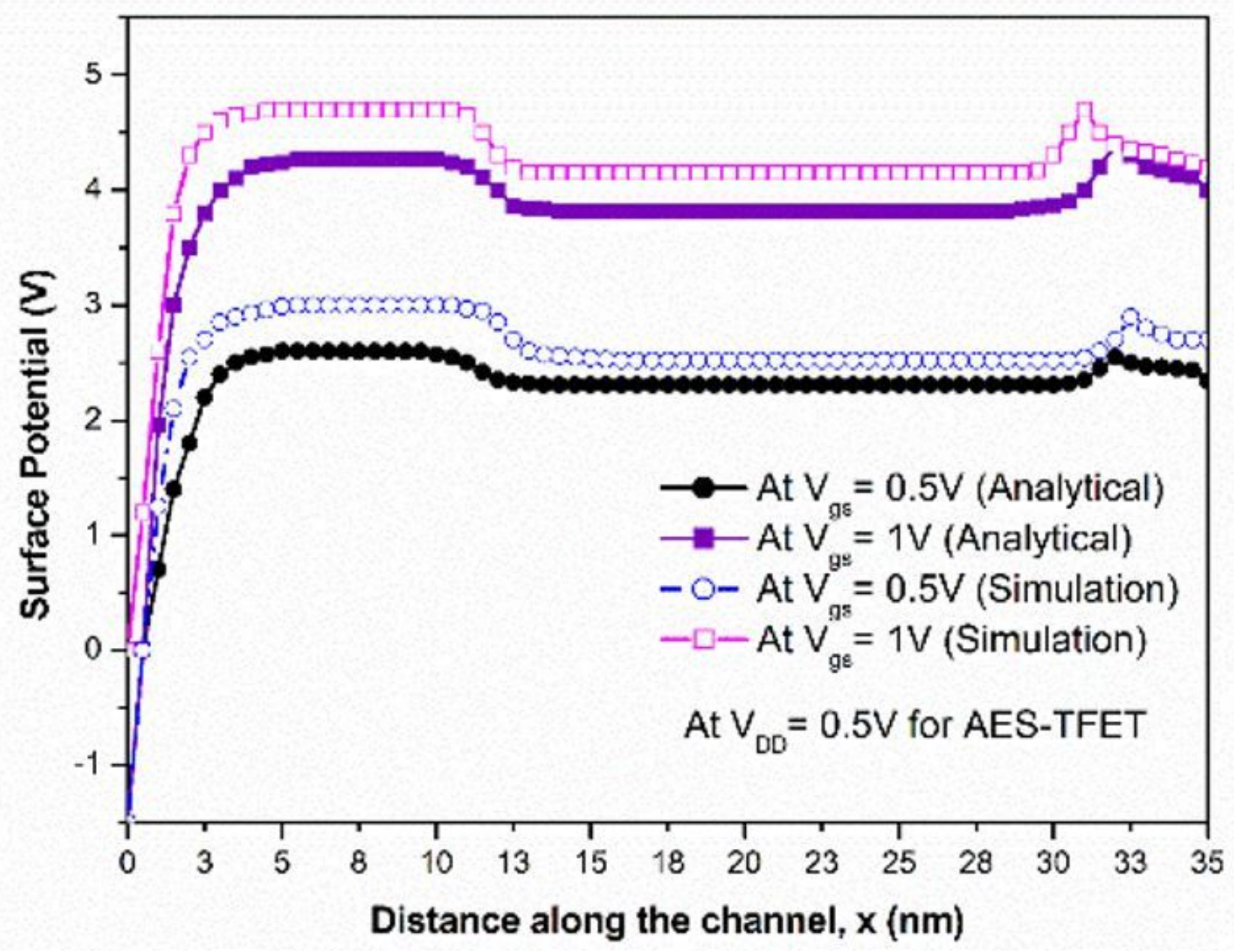

Figure 4

Surface potential distribution along the channel of AES-TFET for different gate-to-source voltages (Vgs) at $\mathrm{VDD}=0.5 \mathrm{~V}$ 


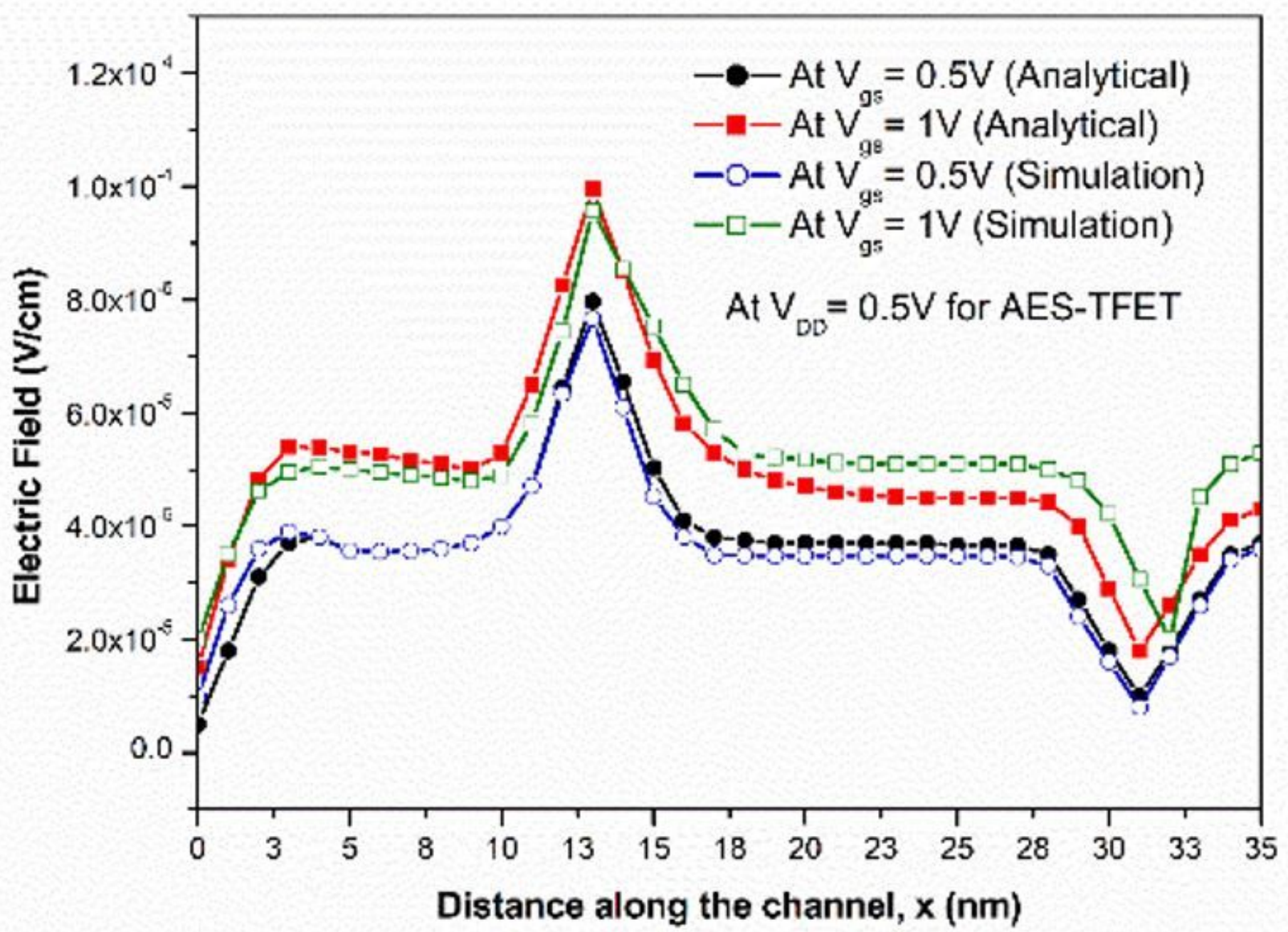

Figure 5

Electric field distribution along the channel of AES-TFET for different gate-to-source voltages (Vgs) at $\mathrm{VDD}=0.5 \mathrm{~V}$ 


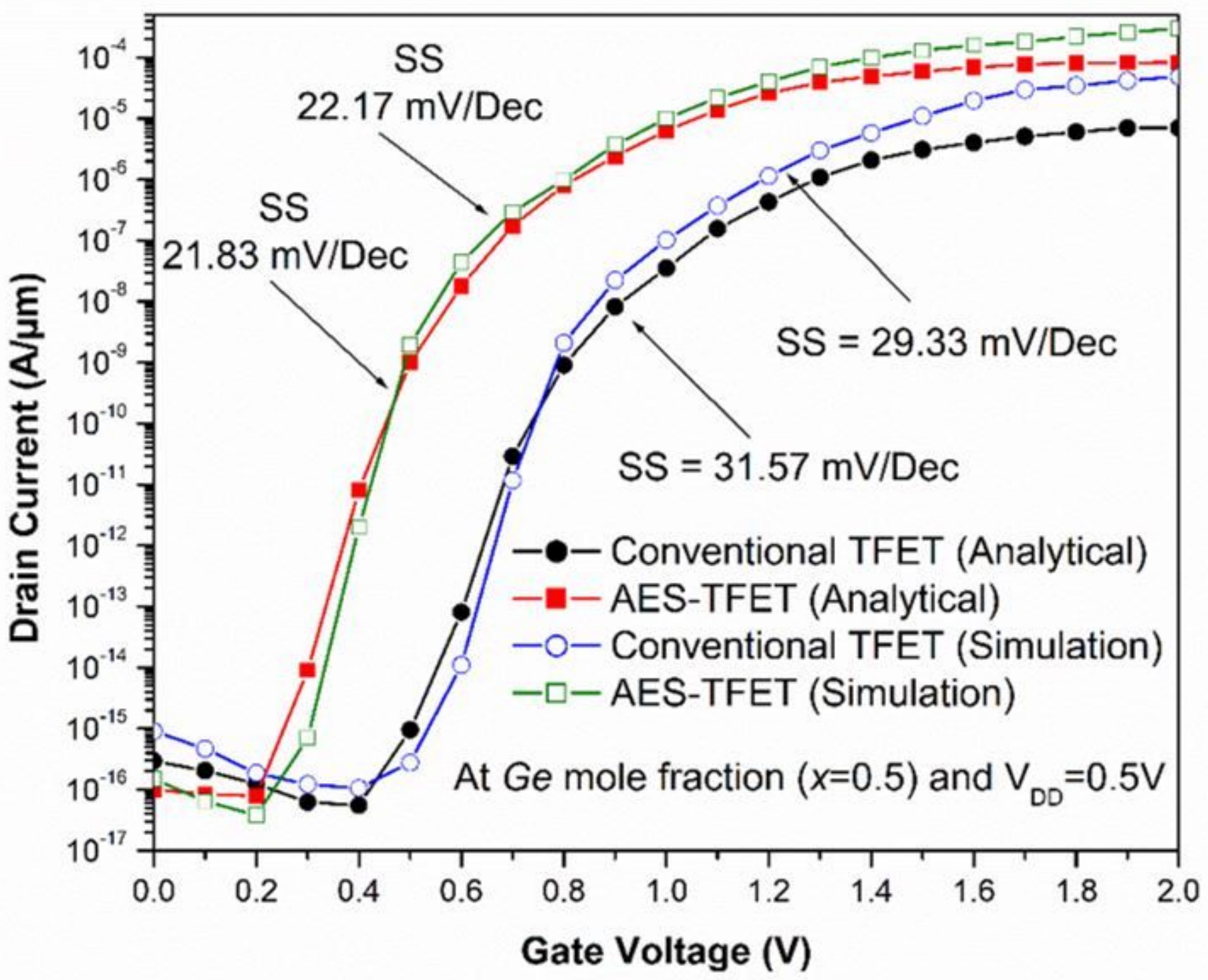

Figure 6

Transfer characteristics (Id vs Vgs) analysis between Conventional TFET and proposed AES-TFET at $\mathrm{VDD}=0.5 \mathrm{~V}$ 


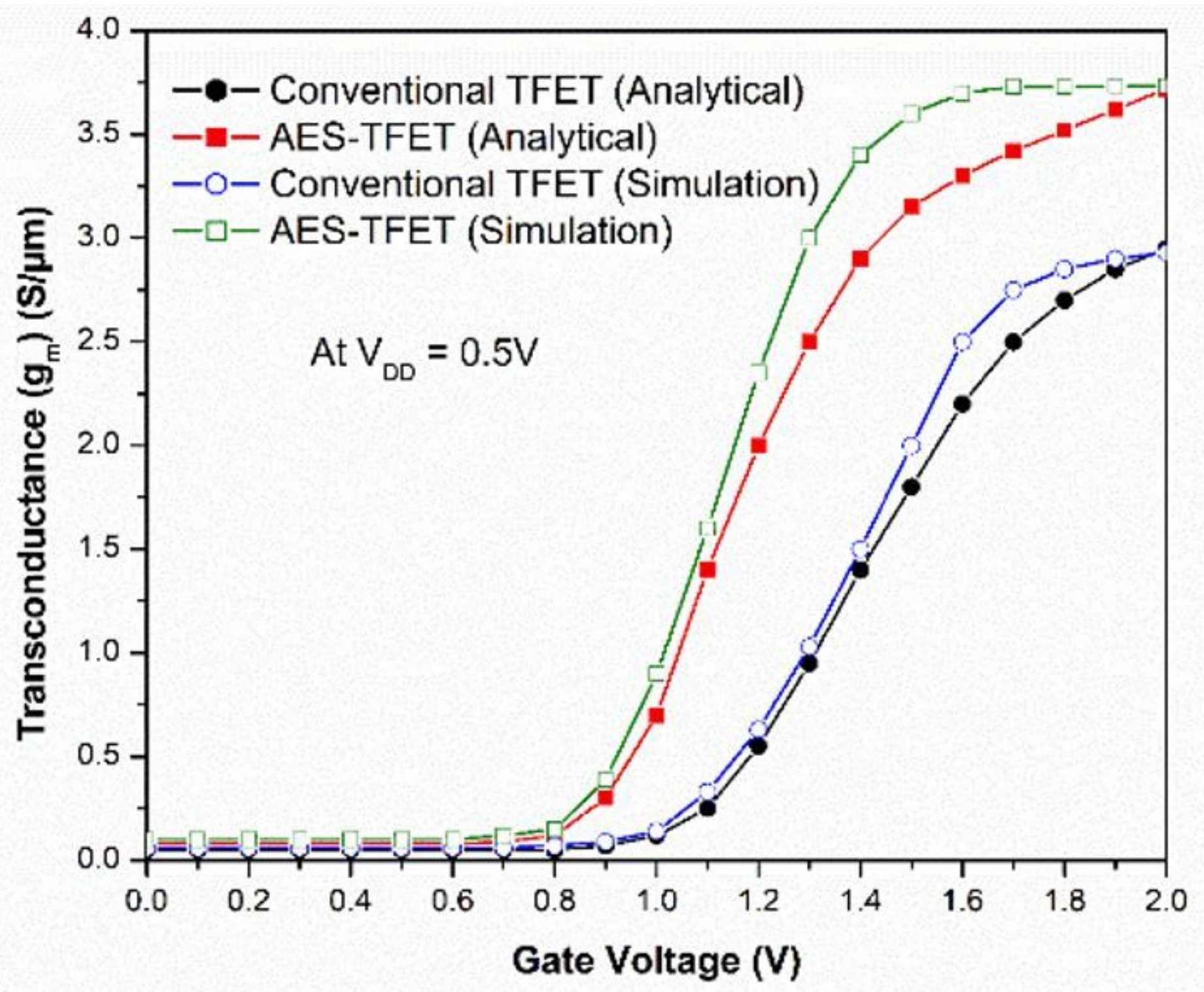

Figure 7

Transconductance (gm vs Vgs) analysis between Conventional TFET and proposed AES-TFET at VDD= $0.5 \mathrm{~V}$

\section{Supplementary Files}

This is a list of supplementary files associated with this preprint. Click to download.

- COVERLETTERSILICON.docx 